\title{
Label Noise Correction and Application in Crowdsourcing
}

\author{
Bryce Nicholson $^{1}$, Victor S. Sheng ${ }^{1}$, and Jing Zhang ${ }^{2}$ \\ 1 Computer Science Department, University of Central Arkansas, United States \\ \{bnicholson 1, ssheng\}@uca.edu \\ 2 School of Computer Science and Engineering, Nanjing University of Science and \\ Technology, China \\ jzhangenjust.edu. cn
}

\begin{abstract}
The important task of correcting label noise is addressed infrequently in literature. The difficulty of developing a robust label correction algorithm leads to this silence concerning label correction. To break the silence, we propose two algorithms to correct label noise. One utilizes self-training to re-label noise, called Self-Training Correction (STC). Another is a clustering-based method, which groups instances together to infer their ground-truth labels, called Clusterbased Correction (CC). We also adapt an algorithm from previous work, a consensusbased method called Polishing that consults with an ensemble of classifiers to change the values of attributes and labels. We simplify Polishing such that it only alters labels of instances, and call it Polishing Labels (PL). We experimentally compare our novel methods with Polishing Labels by examining their improvements on the label qualities, model qualities, and AUC metrics of binary and multi-class data sets under different noise levels. Our experimental results demonstrate that $\mathrm{CC}$ significantly improves label qualities, model qualities, and AUC metrics consistently. We further investigate how these three noise correction algorithms improve the data quality, in terms of label accuracy, in the context of image labeling in crowdsourcing. First, we look at three consensus methods for inferring a ground-truth label from the multiple noisy labels obtained from crowdsourcing, i.e., Majority Voting (MV), Dawid Skene (DS), and KOS. We then apply the three noise correction methods to correct labels inferred by these consensus methods. Our experimental results show that the noise correction methods improve the labeling quality significantly. As an overall result of our experiments, we conclude that $\mathrm{CC}$ performs the best. Our research has illustrated the viability of implementing noise correction as another line of defense against labeling error, especially in a crowdsourcing setting. Furthermore, it presents the feasibility of the automation of an otherwise manual process of analyzing a data set, and correcting and cleaning the instances, an expensive and time-consuming task.
\end{abstract}

\section{Keywords}

noise correction; crowdsourcing; image processing; classification

(C) 2016. This manuscript version is made available under the Elsevier user license http://www.elsevier.com/open-access/userlicense/1.0/ 


\section{Point-to-Point Responses}

\section{Reviewer \#1}

1. I have just a minor observation regarding the label noise generation (in section 4.1), which probably could be enhanced by considering different random generation laws (not only the uniform one). We have also run our noise correction experiments after generating noise from a normal distribution. We include charts depicting the average accuracy, AUC, and model quality of the binary class data sets and multi-class data sets.

\section{Reviewer \#2}

1. The authors should give the readers some concrete information to get them excited about their work. The current abstract only describes the general purposes of the article. It should also include the article's main (1) impact and (2) significance on expert and intelligent systems. We have included the impact and significance of the work in the abstract. They are in the second to last sentence and the last sentence, respectively, of the abstract.

2. Please give a frank account of the strengths and weaknesses of the proposed research method. This should include theoretical comparison to other approaches in the field. We have provided the theoretical and intuitive analysis of strengths and weaknesses of the noise correction methods as Section 3.4.

3. Moreover, I believe that it will make this paper stronger if the authors present insightful implications in a separate paragraph based on their experimental outcomes. We present our implications of both our artificial noise level experiments and our crowdsourcing experiments. The paragraphs containing the implications can be found as the last paragraph of Section 4, and the last paragraph of Section 5, respectively.

4. Finally, please discuss several (say 4-5) solid and insightful future research directions. We discuss future research topics in the last paragraph of Section 6.

\section{Introduction}

A main concern in data preprocessing is ensuring that the data in question is clean. In real-world applications, label noise is an unavoidable phenomenon. Label noise may be the result of many reasons. Labeling error is one of the main causes. It is unfortunate that label noise is so prevalent. However, data practitioners need reliable data for practical applications, such as informational retrieval (Xia, Wang, Sun, \& Wang, 2015), statistical analysis, or machine learning (Zheng, Jeon, Xu, Wu, \& Zhang, 2015). A well-known domain that needs to deal with label noise is crowdsourcing, in which human workers can be hired to label instances.

Crowdsourcing is defined as the distribution of tasks by requesters to non-expert workers in a web-based environment (Brabham, 2008). A popular medium through which requesters crowdsource tasks is Amazon's Mechanical Turk (Kittur, Chi, \& Suh, 
2008). These workers process, categorize, or label the data in ways that a machine cannot. It is sometimes necessary to use human workers for data processing. There is a wealth of data in areas where humans have clear superiority over machines, such as text and image processing.

In (Nowak \& Ruger, 2010), images are annotated via crowdsourcing, and the annotations of expert and non-expert annotators are compared. Annotation can entail one or more labels ascribed to images. This paper will focus only on single-label annotations, which signify the class of each image, or more generally, each instance.

Non-expert annotators have a tendency to err in crowdsourcing tasks. Therefore, it is necessary to acquire annotations from numerous annotators for a single instance. The effectiveness of this practice is shown in (Sheng, Provost, \& Ipeirotis, 2008). Once the requester has obtained multiple labels for each image, he must find a way to integrate the labels, resulting in a single label for each image. This process, called consensus, can be carried out in a variety of ways (Tang \& Lease, 2011).

Even when many annotations have been acquired and consensus has been done, the data will still contain misclassified, or noisy, instances. Fortunately, there are mechanical remedies to the problem of noise, such as noise filters (Khoshgoftaar \& Rebours, 2007) (Devijver, 1980) (Gamberger, Boskovic, Lavrac, \& Groselj, 1999) (Sanchez, Barandela, Marques, Alejo, \& Badenas, 2003) (Ma et al., 2015). The noise reduction technique we will examine in this paper is noise correction, the systematic alteration of instances' classes in an effort to improve data quality.

In this paper, we consider label noise to be a random phenomenon. However, label noise is not always completely random. There exists a phenomenon called adversarial noise, which occurs when a malicious agent is permitted to select labels for a given number of instances (Auer \& Cesa-Bianchi, 1998). Typically, this agent would introduce noise in such a manner as to be particularly difficult to handle.

Although there is a shortage of literature on label noise correction, there is a lot of literature on handling label noise in general. One method of handling label noise is using a noise filter. A noise filter explicitly seeks to eliminate instances from a training set, which are determined by the filter to be mislabeled. Types of noise filters include Multiedit (Devijver, 1980), depuration (Sanchez et al., 2003), Classification Filter (Gamberger et al., 1999), and Iterative Partitioning Filter (Khoshgoftaar \& Rebours, 2007). Noise filters, despite being a generally effective group of methods for handling noise, can pose the drawback of over-cleansing, or filtering too many instances (Frenay \& Verleysen, 2014), such that the remaining instances are not enough to train a good classifier. Another group of methods that implicitly handle label noise, using classification methods that are robust to mislabeled data, are bagging, boosting, and random forests (Dietterich, 2000), Bayesian approaches-e.g., (Gaba \& Winkler, 1992)among many others, which are methods known to perform well even in the presence of noise (Frenay \& Verleysen, 2014). Specifically, one work focuses on minimizing the risk incurred from label noise to create an unbiased learning algorithm, assuming that the noise levels are known (Natarajan, Dhillon, Ravikumar, \& Tewari, 2013). However, according to our knowledge, there is only one method to correct noisy labels (belonging to a discrete set) - that is, to identify each mislabeled instance and to correct it according to what a correction method deems its most likely ground-truth label (i.e., its true 
class) to be. This method in literature is called Polishing. Polishing aims to change attribute values of instances as well as labels (Teng, 1999). It has been observed that the effects of label noise are much more detrimental to classification than that of attribute noise (Xu \& Wunsch, 2005) (Saez, Galar, Luengo, \& Herrera, 2014) (Xia, Wang, Sun, Liu, \& Xiong, 2014) (Xia, Wang, Sun, \& Wang, 2014). We seek to tackle this problem first by considering only label noise.

As a side note, there is another method for noise correction (Song, Wang, Zhang, Sun, \& Yang, 2015), but it concerns textual "spectral" labels, while we consider only the discrete case. Because of the lack of literature on discrete label noise correction and the data practitioner's need to improve data quality, it is necessary for us to conduct foundational research on this topic and to open the doorway for future contributions.

In Section 2, we begin by discussing three consensus methods to integrate workers' labels in crowdsourcing. In Section 3, we propose two correction methods (STC and $\mathrm{CC}$ ), and another method (PL) we adapt to compare with our methods. In Section 4, we evaluate these three methods in their ability to improve label quality, model quality, and AUC on binary class data sets and multi-class data sets under artificial levels of noise. In Section 5, we test the correction methods' ability to improve data accuracy on real-world crowdsourced data, derived from image classification tasks (Wen, Shao, Xue, \& Fang, 2015). In Section 6, we conclude this paper with several remarks.

\section{Consensus Methods}

Consensus is an important inference process to integrate multiple noisy labels to a single label. Kamar, Hacker, and Horvitz (2012) define consensus as a process to "idenify a hidden state of the world by collecting multiple assessments from human workers" (Kamar, Hacker, \& Horvitz, 2012). Consensus makes it possible for only slightly accurate labelers to collectively contribute to a very accurate labeling of instances. There are several well-known inference algorithms, such as majority voting, Dawid Skene (Dawid \& Skene, 1979), and KOS (Karger, Oh, \& Shah, 2011), which will be described in this section.

Majority voting is the simplest method of inferring an integrated label. It selects the most frequent label among the group of noisy labels as the integrated label. Clearly, this naive method can be greatly improved upon.

Dawid and Skene (1979) applied Expectation Maximization (EM) to conduct consensus from a set of noisy labels (Dawid \& Skene, 1979). In effect, their consensus algorithm iteratively estimates the classes that are maximally likely to be the true classes, then uses that information to estimate the error rate of each worker and the class distributions, and repeats those steps until a convergence is reached. Thus, the process yields a final estimation of the true class of each instance. Throughout this paper, we will refer to this consensus algorithm as DS. DS was successfully used in an experiment to estimate the fitness of patients, achieving a very high accuracy of $96 \%$ (Wilson, Williams, Baskett, Bennett, \& Skene, 1980).

Karger et al. (2011) developed a consensus algorithm that utilizes the concept of worker messages and task messages to arrive at a consensus for each task's correct answer, or true class (Karger et al., 2011). It works by constructing a graph of workers 
and tasks, where an edge from a worker to a task denotes that the task is assigned to the worker. A worker message is directed from a worker to a task and contains information about how reliable the worker is with respect to that task. Likewise, task messages are directed from tasks to workers. The algorithm uses task messages to update worker messages, and vice versa, in an iterative manner. The true classes are then estimated from the information contained in the task messages. We refer to this algorithm as KOS in the remainder of this paper.

Although consensus can often produce data sets with high label accuracy, the inferred labels still contain noise. It is beneficial to attempt to correct the labels through noise correction. Noise correction entails using an algorithm that determines which instances are likely to be mislabeled and relabels them. The next section discusses three noise correction algorithms we use in our experiments.

\section{Label Noise Correction Methods}

We define three methods to correct label noise in this section, which we use in our experiments in the next section. We first adapt Teng's method (Teng, 1999) such that it only corrects labels, preserving as much of her Polishing algorithm as possible. Then, we will introduce our proposed methods, Self-Training Correction (STC) and Clusterbased Correction (CC).

\subsection{Polishing Labels}

As we discussed in Section 1, according to our knowledge, Teng's method (Teng, 1999) seems to be the only noise correction method available in literature. Unfortunately, it also focuses on attribute noise correction, which is not considered in this paper. We remedy this by simplifying the algorithm such that it only focuses on improving the label quality of each data set. It works by taking a single classification algorithm and building ten different models, separating the data set into ten folds on which to build each model. Each instance in the set is then given a vote, or a guess as to what its ground-truth label is, from each model, resulting in ten votes per instance. The label that receives the most votes is ascribed to the instance. If there is a tie and the original label is involved in the tie, the original label is kept. However, if there is a tie and the original label is not involved in the tie, the instance's new label is randomly selected from the labels involved in the tie.

We acknowledge that this implementation is a vast simplification of the author's original algorithm, yet it is justified because the domain of our problem does not include altering attribute values. Since she called this method Polishing, we refer to our adaptation as Polishing Labels (PL).

\subsection{Self-Training Correction}

The problem of label noise correction is not unlike that of using a set of labeled data to infer labels of originally unlabeled data, a process known as self-training, described by (Triguero, Saez, Luengo, Garcia, \& Herrera, 2014). Extending the notion to apply 
to label noise correction is as simple as considering the labeled data in the self-training paradigm as the pool of data remaining after a noise filter has discarded some instances from a data set, which are determined to be noisy. The unlabeled data is therefore analogous to the set of discarded instances, and the task of correcting noisy data becomes easily extendable to the self-training paradigm.

In specific, the STC algorithm first uses a noise-filtering algorithm on a data set to generate a noisy set and a clean set. Then, the algorithm builds a model from the clean set and uses that to calculate the confidence that each of the instances from the noisy set is mislabeled. The noisy instance with the highest calculated likelihood of belonging to some class that is not equal to its current class is relabeled to the class that the classifier determined is the instance's most likely true class. The relabeled instance is then added to the clean set, and the process is repeated until some proportion of noisy instances is relabeled and added to the clean set. STC is flexible in that the user can specify what proportion of noisy instances he wants to correct.

With that in mind, we take inspiration from the self-training algorithm of (Triguero et al., 2014) and develop a noise correction algorithm we call STC.

\subsection{Cluster-based Correction}

We propose a novel method for label noise correction, which is based on clustering, a popular unsupervised learning technique (Jain, Murty, \& Flynn, 1999). The beauty of clustering in a noisy scenario is that clusters are formed independently of instances' labels-regardless of the noise level of a data set, the same clustering algorithm (with the same parameters) will cluster the data set in exactly the same way. This is completely different from the previous two methods, PL and STC. Both PL and STC depend on the label quality of the original data set.

Our Cluster-Based Correction method (denoted as CC) executes one or more clustering algorithms on a training set several times, giving the same set of weights to all instances in each cluster based on the distribution of ascribed labels in that cluster and the cluster's size. The weights favor the class where the majority (or plurality) of instances belong to that class. After the data set has been clustered several times, weights obtained from each cluster are summed for each instance, and each instance is given the label corresponding to the maximum weight.

One Implementation of Cluster-based Correction In this paper, we propose a single implementation of $\mathrm{CC}$, acknowledging that $\mathrm{CC}$, instead of embodying just one algorithm, is the framework for a family of algorithms, all of which conform to the above description. Its pseudo-code is shown in Algorithm 1.

The label vector $\mathbf{y}$ contains all the ascribed labels for the instances in the data set $X$, and the label set $Y$ contains all possible values for the labels in $\mathbf{y}$.

Lines 1 - 4 in Algorithm 1 work to count the distribution of ascribed labels in the data set. This information will be utilized to calculate the label weights for each instance, shown in Algorithm 2. Lines 5 - 14 in Algorithm 1 perform all required clustering algorithms. In our case, we use $k$-means clustering, varying $k$ from two to roughly half the number of instances in the set (Line 6). This process should yield lots of large 
and small clusters, which adds to the diversity of our clusters. Line 11 calculates the weights for each instance in each cluster. Finally, Lines 15 - 18 use the totaled weights to perform correction.

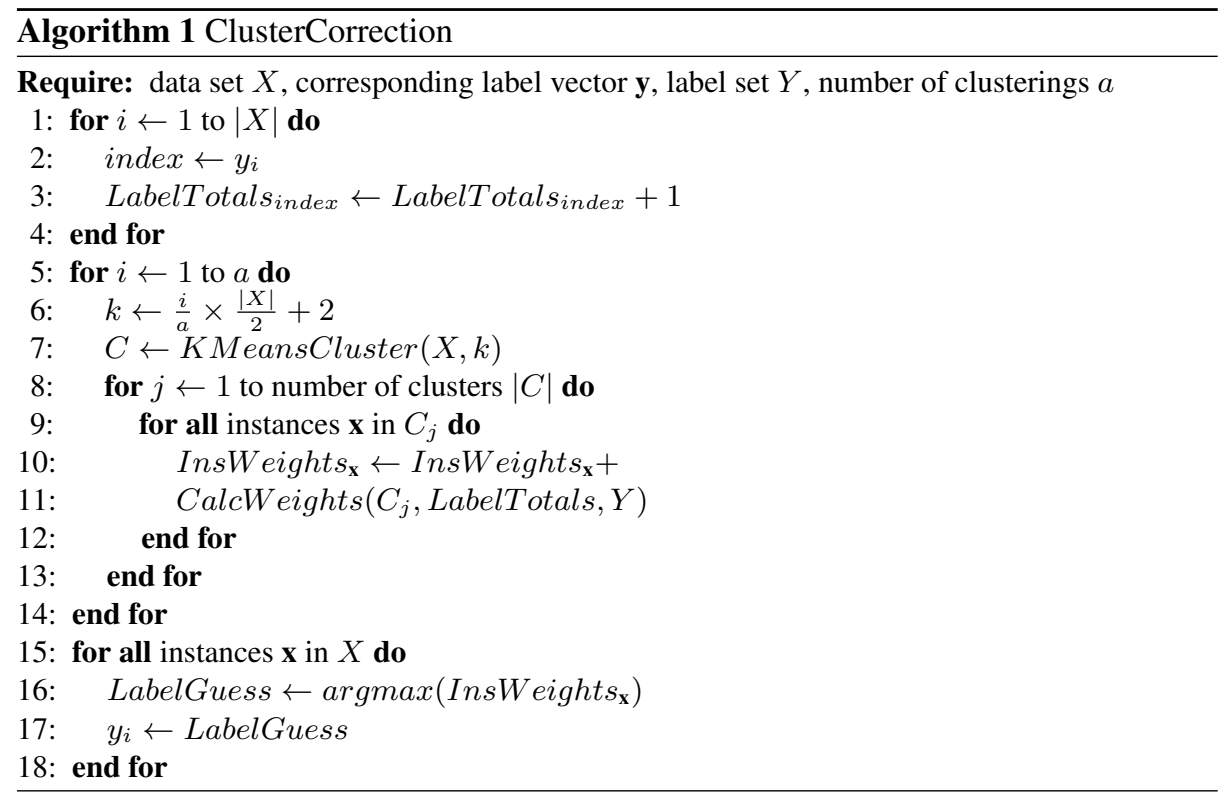

The process of calculating weights is detailed in Algorithm 2.

Note that the multiplier (Line 5 in Algorithm 2) works to give more magnitude to the larger clusters, so that the maximum multiplier, obtained from a cluster of 100 instances, is 2 . We do this so that very big clusters do not eclipse the smaller ones in significance. $\frac{d_{i}-u_{i}}{v_{i}}$ calculates the difference between the expected proportion $u_{i}$ (i.e., $\frac{1}{|Y|}$, where $|Y|$ is the number of classes) of instances of class $i$ in a cluster with no useful information and the actual proportion $d_{i}$ of instances of class $i$ in the cluster, scaled by the proportion $v_{i}$ of instances of class $i$ in the data set.

At first glance, it seems that using only the $k$-means clustering algorithm may be a short-sighted implementation choice. Can only considering one type of clustering algorithm yield sufficient diversity? While it may be true that more cluster diversity can be obtainable by conferring with multiple types of clustering algorithms, a sufficient degree of diversity can be achieved with $k$-means alone. This is ensured by $k$-means's tremendous dependence on its initial centroid generation (Xu \& Wunsch, 2005). Different from previous work, we strive to increase the diversity of our clusters by varying the number of initial centroids $k$. The effectiveness of considering a range of $k$-values in $k$-means clustering is validated in (Likas, Vlassis, \& Verbeek, 2003), where the authors concluded that this technique "will always provide sensible clustering solutions." Therefore, combining $k$-means's initial centroid sensitivity with a varying number of initial centroids $(k)$ is a recipe for cluster diversity. 


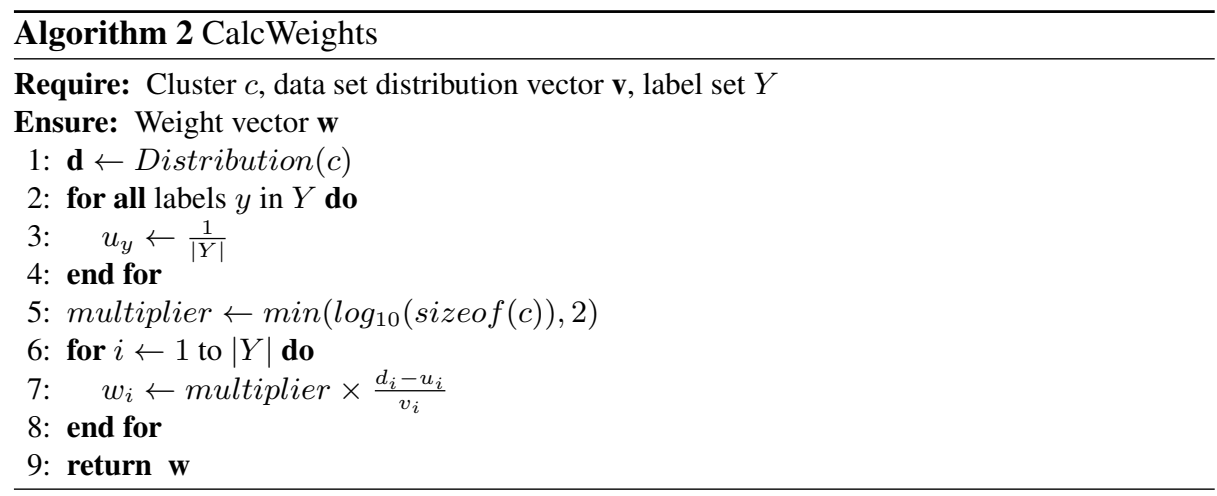

\subsection{Theoretical Comparison of the Correction Methods}

Before we compare the three noise correction methods experimentally, we do some theoretical analysis to compare them. We make some assumptions about conditions that are commonly true in real-world applications, and describe the performance of the noise correction methods when those assumptions hold.

In our analysis of the noise correction methods, we use the notation $I$ to refer to the set of instances in our data set, $r$ to refer to the proportion of instances that are noisy, $c$ to refer to the classifier used in the noise correction process, and $\bar{S}$ to refer to the number of elements of some set $S$.

Polishing Labels Polishing Labels starts by partitioning the instance set $I$ into ten subsets of equal size $I_{1}, I_{2}, \cdots I_{10}$. It uses a classifier $c$ to build a model on each partition, creating ten (initialized) classifiers, say $c_{1}, c_{2}, \cdots c_{10}$. Let $p$ be the probability that, for any $i$ in $I$, some classifier $c_{k}$ correctly labels $i$. We can assume that $p$ is the same regardless of which classifier (i.e., which model $c$ built from a partition of $I$ ) is classifying $i$, because given a large or predictable enough data set, only considering one tenth of the data set to build a model will only minimally affect classification.

Suppose there is $r$ proportion of noise in the data set. Because the probability of the classifier correctly labeling an instance is influenced by the noise level of the data set, let $p_{r}$ be the probability of some classifier $c$ correctly labeling an instance, given there is $r$ noise in the data set.

Each of the ten classifiers classifies every instance of all other partitions. Therefore, each instance has nine labels. Polishing label gives each instance the class value that had the majority of votes from the classifiers. So the probability that the majority of labels of an instance $i$ are correct is given by

$$
\sum_{m=5}^{9}\left(\begin{array}{c}
9 \\
m
\end{array}\right) \times p_{r}^{m} \times\left(1-p_{r}\right)^{9-m}
$$


Self-training Correction Self-training Correction uses some noise filter, $f$, to separate the instance set $I$ into $I_{c}$, the clean instance set, and $I_{r}$, the noisy instance set, according to the filter. Because most real-world noise filters use a classifier (e.g., Iterative Partitioning Filter, Classification Filter, etc. (Gamberger et al., 1999)), let us assume that $f$ uses a classifier, $c$. Then let $p_{c, r}$ be the probability that classifier $c$, having built a model on $I_{c}$, correctly classifies some instance of $I_{r}$. Further, let us assume that for each class that $c$ assigns to some $i$ in $I_{r}$, there is a corresponding confidence value, $d$, such that when $c$ correctly classifies $i, d$ is uniformly distributed on the interval $(.5,1]$. Similarly, when $c$ incorrectly classifies $i, d$ is also uniformly distributed on the interval $(.5,1]$. Since Self-training correction operates by choosing the instance in $I_{r}$ with the highest $d$-value and transferring the instance to $I_{c}$, it follows that the probability that the instance in $I_{r}$ that was given the highest confidence value was correctly classified is the same as $p_{c, r}$, because there are $p_{c, r} \times \bar{I}_{r}$ correctly classified instances with confidence values uniformly in $(.5,1]$, and $\left(1-p_{c, r}\right) \times \bar{I}_{r}$ incorrectly classified instances with confidence values uniformly in $(.5,1]$. For any instance $i$ in $I$, the probability that it is clean is based on whether (1) it was clean before filtering took place, and after filtering $i$ was in $I_{c}$, or (2), if it was in $I_{r}$, whether it was selected in one of the iterations as the highest confidence instance and was labeled correctly then. So let $p_{f}$ be the probability that an instance in $I$ was put in $I_{c}$ if the instance was clean, or put in $I_{r}$ if it was noisy. Thus, the probability of an instance $i$ being correctly labeled after Self-training Correction can be mathematically expressed by

$$
\frac{p_{f} \times \bar{I}_{c}+\sum_{k=1}^{t} p_{c_{k}, r_{k}}}{\bar{I}}
$$

where $p_{c_{k}, r_{k}}$ is the probability that $c$ correctly classifies an instance of $I_{r}$ given it has built a model on $I_{c}$, in the $k$ th iteration, and $t$, the threshold, is the number of instances to move from $I_{r}$ to $I_{c}$ when they are initially formed as a result of filtering. Keep in mind that $I_{r}$ and $I_{c}$ are dynamically changing at every iteration, when the most confident instance of $I_{r}$ is moved to $I_{c}$.

Cluster-based Correction Cluster-based correction uses a clusterer, $c l$, and initializes it $n$ times with different random seeds to create $n$ distinct (initialized) clusters $c l_{1}, c l_{2}$, $\cdots c l_{n}$.

Suppose the instances in $I$ have a spatial relation factor $s$; that is, given an arbitrary number of clusters, $s$ is the probability that if two instances $i_{1}$ and $i_{2}$ in $I$ share the same cluster, then they share the same class value. Each clusterer described earlier will cluster the instances, creating $n$ sets of clusters, where the number of clusters in each cluster set is arbitrary. Thus, every instance $i$ will find itself ( $n$ times) in a cluster with some number of neighbors, say $g_{i, k}$, the number of neighbors of instance $i$ has as a result of the clustering of clusterer $k$. Also, $c_{i, k}$ is the cluster generated by the $k$ th clusterer that instance $i$ belongs to. Clearly, the number of instances in the cluster that $i$ belongs to is $g_{i, k}+1$. Finally, suppose there is some weight function $w$ that weights a cluster based on its size, to give more weight to clusters with larger sample size. Then, given a noise level $r$, the probability of an instance $i$ being clean as a result of Cluster-based 
correction is

$$
\sum_{k=1}^{n} s \times r \times w\left(c_{i, k}\right)
$$

Comparison Even given the theoretical assumptions above, it is impossible to determine which correction method is theoretically superior. Both Polishing Labels and Self-training Correction make use of a classifier, but PL's classifier builds a model on a data set with a constant noise level, while STC's model deals with a dynamic noise level because it is constantly altering the clean set as it adds previously noisy instances. Cluster-based Correction, on the other hand, does not use a classifier at all but a clusterer; the two cannot be theoretically compared.

PL is the simplest of the three correction methods. Its simple use of a classifier to create multiple labelers makes its effectiveness entirely dependent on how well the classifier it uses performs on the data set. This implies that it is straightforward to understand why PL performs the way it does on a certain data set, but its lack of complexity makes it inferior on data sets that require more sophisticated methods. Its simplicity also implies a faster run time.

STC is the more advanced of the correction methods that use a classifier. It uses the classifier to rebuild a model every time a noisy instance is relabeled and added to the set of clean instances. Conceptually, this would be thought to result in a model that constantly increases in quality as more and more instances are cleaned and used in the model. However, the opposite can also occur; initial mistakes can be made that worsen the model, which can lead to more frequent mistakes and overall a bad performance for the noise correction method. Again, the performance of STC is dependent on the classifier in use, but the classifier is not applied in such a straightforward manner as it is in PL. Because it has to (1) build a model every time it reclassifies and adds an instance to the clean set, and (2) classify every instance in the noisy set before it selects the one with highest confidence, its run time is significantly longer than PL.

$\mathrm{CC}$ is the odd one out among the noise correction methods. It uses clustering instead of classifiers. Thus, it cannot be compared to the others theoretically. Similarly to PL (and STC), though, its performance does depend on how well the method-in this case, clustering-fits the data set. That is, CC's performance is directly proportional to how spatially related the data are. If clustering is a poor fit for the data, CC may harm the data quality. Depending on how $\mathrm{CC}$ is implemented, it could require a large number of clusterers to run. In our experiments, for example, we run 200 clusterers, some of which may create hundreds or thousands of clusters. This can imply massive amounts of computation time, likely much more than the other two, but for the goal of revealing spatial associations among the data, if they exist, it does well.

\section{Artificial Noise Level Experiments}

In this section, we describe our experimental setup using several artificial levels of noise, and provide our experimental results, comparing the three methods in terms of label quality, model quality, and AUC. In multi-class scenarios, AUC is calculated using a pairwise average AUC metric over each pair of classes. 


\subsection{Setup}

We use WEKA (Hall et al., 2009) in both our classification and clustering tasks for the following experiments. We carry out our experiments by taking clean data sets and artificially injecting $p$ noise into them, generating the noise based on uniform and normal probability distributions. That is, for the uniformly generated noise, given a data set $X$, we select $p \times|X|$ instances at random, assigning them a different label (randomly, when there is more than one alternative, as in multi-class problems). For the normally generated noise, we select $p \times|X|$ instances based on the mean and variance of each of their features, more likely choosing instances whose features were generally close to the mean. We use nine noise levels $p$ to investigate the performance of the three algorithms. That is,

$$
p \in\{0.05,0.10,0.15,0.20,0.25,0.30,0.35,0.40,0.45\} .
$$

Polishing Labels As our classifier for PL, we used WEKA's C4.5 decision tree implementation, J48.

Self-Training Correction For our implementation of STC in these experiments, we run Classification Filter on each data set using J48, and set the number of folds for validation at 10 . The STC algorithm itself also uses J48 as its classifier.

Cluster-based Correction Our implementation of CC is mostly detailed in the pseudocode shown in Algorithms 1 and 2. One extra detail is that we use 200 iterations of WEKA's SimpleKMeans algorithm.

Data Sets To investigate the performance of the three label correction methods on binary and multi-class scenarios respectively, we choose half our data sets to be binary data sets and the other half to be multi-class data sets. Therefore, we will conduct our experiments on 12 binary class data sets and 12 multi-class data sets. Their characteristics are shown in Table 1 and Table 2, respectively. The latter four columns of both Table 1 and Table 2 display the number of categorical (or nominal) attributes, the number of numerical attributes, the number of instances, and the number of classes in each data set, respectively.

\subsection{Experimental Results}

In evaluating the performance of the three label noise correction methods, label accuracy, resulting model quality, and AUC are all used as the measurement in our experiments. Label quality is defined in terms of accuracy (i.e., the proportion of instances whose ascribed label and true label are the same). Model quality is defined as the classification accuracy obtained by training a classifier on the corrected data set and performing cross-validation. In our case, we use WEKA's SMO Support Vector Machine classification algorithm (Hall et al., 2009), with 10 folds for cross-validation. Finally, AUC represents the Area Under the Curve of the Receiver Operating Characteristic 
(ROC) curve. AUC reflects how well each class was predicted, so that if a minority class is misclassified frequently, it would not harm label quality as much as it would harm AUC. We will discuss the effectiveness of these three methods in terms of label quality improvement, model quality improvement, and AUC improvement.

\begin{tabular}{|l|l|l|l|l|}
\hline Data Set & $\begin{array}{l}\text { Cat. } \\
\text { Atts. }\end{array}$ & $\begin{array}{l}\text { Num. } \\
\text { Atts. }\end{array}$ & Insts. & Classes \\
\hline breast-w & 0 & 9 & 699 & 2 \\
\hline colic & 20 & 7 & 368 & 2 \\
\hline credit-a & 9 & 6 & 690 & 2 \\
\hline credit-g & 13 & 7 & 1000 & 2 \\
\hline diabetes & 0 & 8 & 768 & 2 \\
\hline heart-stat-log & 0 & 13 & 270 & 2 \\
\hline hepatitis & 13 & 6 & 155 & 2 \\
\hline ionosphere & 0 & 34 & 351 & 2 \\
\hline kr-vs-kp & 36 & 0 & 3196 & 2 \\
\hline labor & 8 & 8 & 57 & 2 \\
\hline mushroom & 22 & 0 & 8124 & 2 \\
\hline sonar & 0 & 60 & 208 & 2 \\
\hline
\end{tabular}

Table 1: Experimental binary class data sets.

The experimental results of the three methods are shown in figures. On a figure, the horizontal axis represents the label noise level $p$, and the vertical axis represents the respective metric (i.e. accuracy, model quality, or AUC) of the three methods. In order to show the improvement, we have a dashed line to represent the baseline metrics in each figure. For the accuracy figures, each dashed line bisects each figure. For example, the line contains the point $(20,0.80)$. This signifies that at $20 \%$ noise, i.e., $p=0.2$, the baseline label quality is 0.80 . The baselines for model quality and AUC vary more, however. On each figure, the results for Cluster-based correction is shown in blue, the results for Self-Training Correction is shown in red, and the results for Polishing Labels is shown in green.

The area above the baseline signifies improvement, and the area below the baseline denotes decline. The vertical distance between each point in the curve of each method and the baseline shows how much improvement the method has achieved. The higher the vertical distance, the greater the improvement is.

We have results using both uniform and normally generated noise. We show results for all data sets using uniform distribution, including the average overall results and averages for both binary class and multi-class scenarios. However, for normally generated noise, we show only the average results (overall and binary/multi-class). If the figure does not specify the type of noise generation, it is uniform; otherwise, the figure's title will specify that the noise was generated normally. 
Binary Class Data Sets The influence on accuracy (i.e., label quality) of each label noise correction algorithm is shown in Figure 1 for all binary data sets in our experiments. Figure 1 shows that the three label correction methods improve the label quality on most data sets. In all the experimental label noise levels in the interval $[0.05,0.45]$, the three methods significantly improve the label qualities on two data sets (breast- $W$ and mushroom). On most of the remaining experimental data sets, all three methods improve their label qualities after the label noise reaches a certain level. Among the three methods, CC performs the best, followed by STC, in terms of accuracy.

\begin{tabular}{|l|l|l|l|l|}
\hline Data Set & $\begin{array}{l}\text { Cat. } \\
\text { Atts. }\end{array}$ & $\begin{array}{l}\text { Num. } \\
\text { Atts. }\end{array}$ & Insts. & Classes \\
\hline autos & 10 & 15 & 205 & 7 \\
\hline balance & 0 & 4 & 625 & 3 \\
\hline cars & 6 & 0 & 1728 & 4 \\
\hline heart-c & 7 & 6 & 303 & 5 \\
\hline heart-h & 7 & 6 & 294 & 5 \\
\hline iris & 0 & 4 & 150 & 3 \\
\hline lymph & 15 & 3 & 148 & 4 \\
\hline segment & 0 & 19 & 2310 & 7 \\
\hline vehicle & 0 & 18 & 846 & 4 \\
\hline vowel & 3 & 10 & 990 & 11 \\
\hline waveform & 0 & 40 & 5000 & 3 \\
\hline zoo & 16 & 1 & 101 & 7 \\
\hline
\end{tabular}

Table 2: Experimental multi-class data sets.

Figure 2 shows the model quality improvement of each correction method on all the binary class data sets. On most data sets, STC and CC improve model quality from the baseline (e.g. kr-vs-kp, mushroom, sonar). As with accuracy, PL performs worst in terms of model quality. We find that PL harms model quality on the majority of the binary data sets (e.g. credit-a, labor, $k r$-vs-kp). We see again that STC and CC are both very competitive with each other in terms of model quality improvement, while PL performs the worst. 

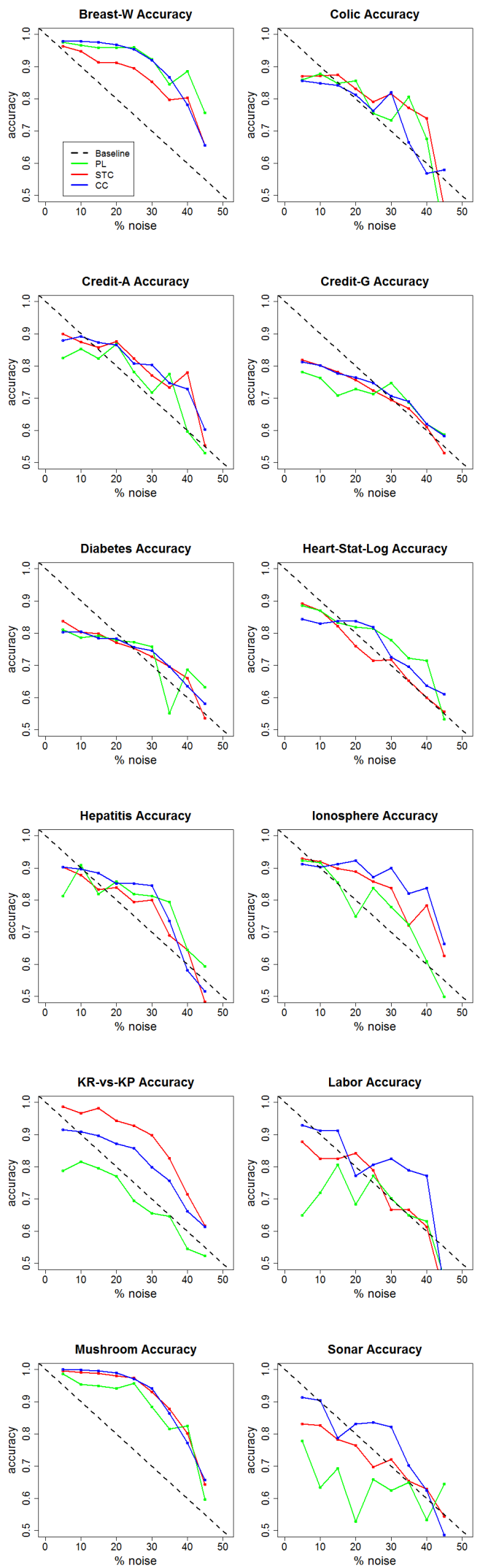

Fig. 1: Accuracy results on binary class data sets. 

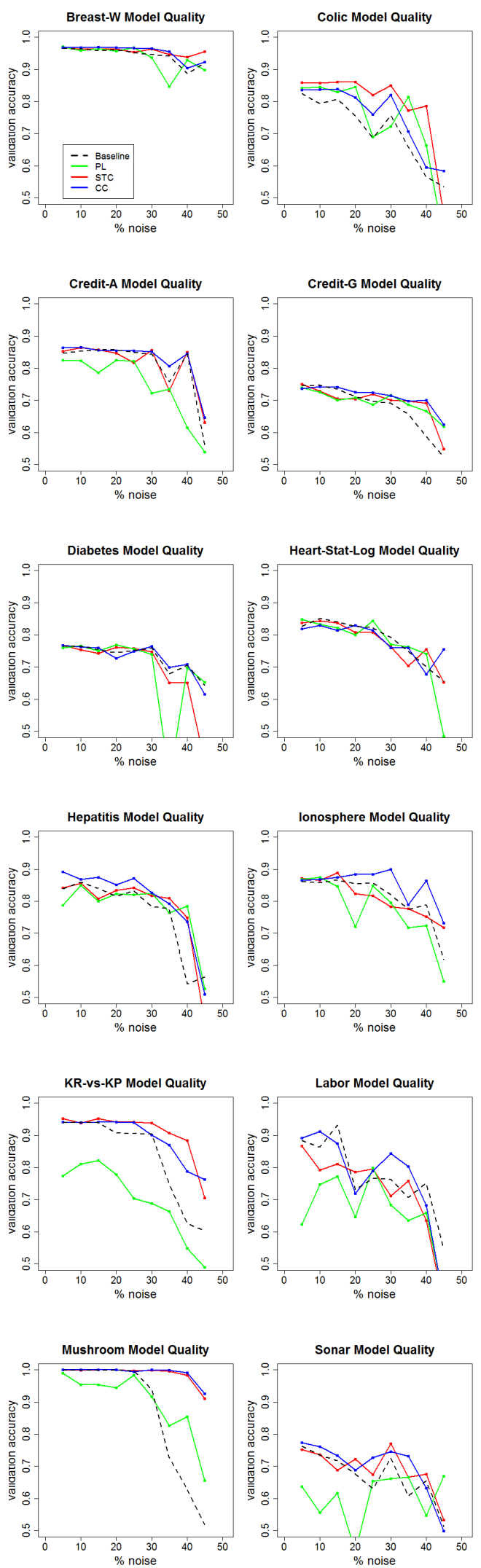

Fig. 2: Model quality results on binary class data sets. 

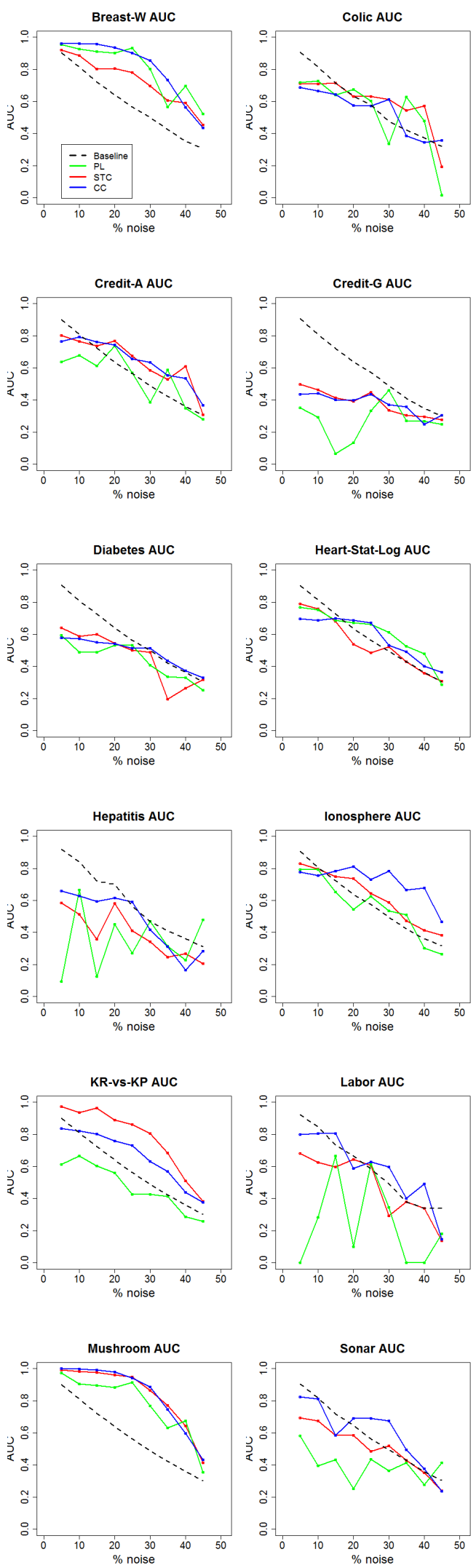

Fig. 3: AUC results on binary class data sets. 


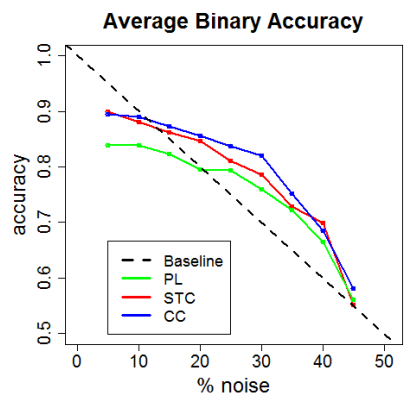

Fig. 4: Average accuracy results on binary class data sets

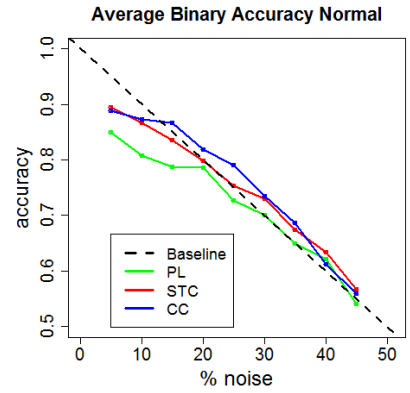

Fig. 5: Average accuracy results on binary class data sets using normal distribution to generate noise

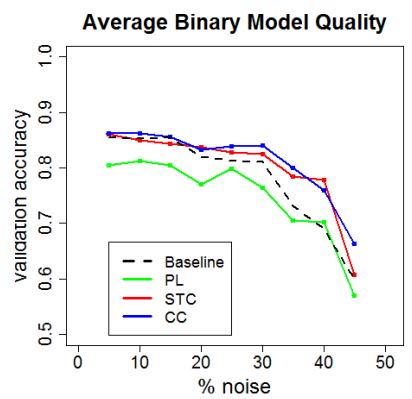

Fig. 6: Average model quality results on binary class data sets 


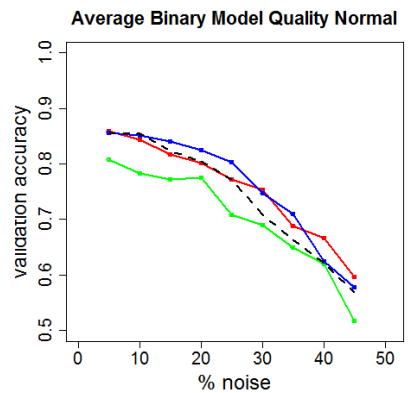

Fig. 7: Average model quality results on binary class data sets using normal distribution to generate noise

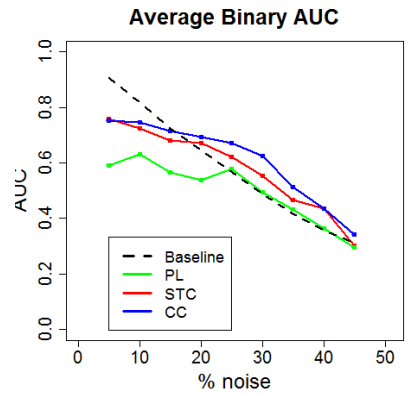

Fig. 8: Average AUC results on binary class data sets

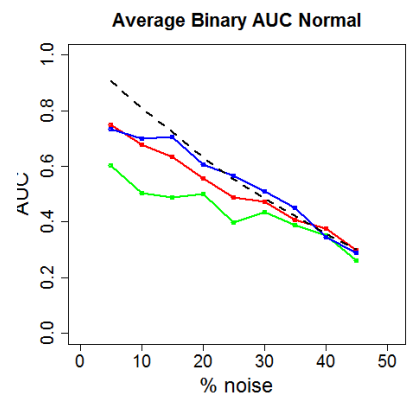

Fig. 9: Average AUC results on binary class data sets using normal distribution to generate noise 
The AUC improvement of each method on each binary class data set is shown in Figure 3. On most of the data sets, we see CC improve AUC from the baseline after the noise reaches a certain threshold (e.g. credit-a, diabetes, heart-stat-log, $k r$ - vs- $k p$ ). On the data sets mushroom and breast-w, all three methods improve AUC under all noise levels.

To summarize the performance of the three methods better, we have the average label quality improvement results over the 12 binary data sets. Figure 4 shows the results using uniform noise generation, and Figure 5 shows the results using normal noise generation. From Figure 4, we can see that when the label noise level $p>0.10$, CC improves the label quality. When the label noise level $p>0.15$, STC can improve the label quality, and when $p>0.20$, PL can finally improve the label quality. Among the three label correction methods, CC outperforms both STC and PL in the experimental label noise range except at $p=0.05$ and $p=0.40$, where STC is only slightly superior. CC dominates both STC and PL on average. PL is only competitive with STC and CC when the noise level $p \geq 0.40$. Interestingly, Figure 5 shows us the accuracy improvement after injecting normally generated noise, which has an almost identical result as Figure 4, except that the accuracy improvement is not as pronounced in Figure 5 as it is in Figure 4.

The average model quality improvement results on the twelve binary class data sets are shown, with the results from uniformly generated noise in Figure 6 and the results from normally generated noise in Figure 7. CC is the only correction method that does not harm model quality on average for any of the noise levels. For all noise levels, CC greatly outperforms PL, and on most noise levels, CC outperforms STC as well. STC is a close second to CC, though, but PL is clearly the worst. PL almost always harms model quality on average.

Figure 8 and Figure 9 display the average AUC improvements of all three correction methods on the binary class data sets, using uniformly and normally generated noise, respectively. For the noise levels $p>0.15$, both STC and CC improve AUC on average, with CC showing superior improvements to STC. For the noise levels $p>0.20$, PL can leave AUC approximately the same as it was before correction on average, but cannot really improve AUC. To conclude, CC is the superior method in terms of AUC improvement on average.

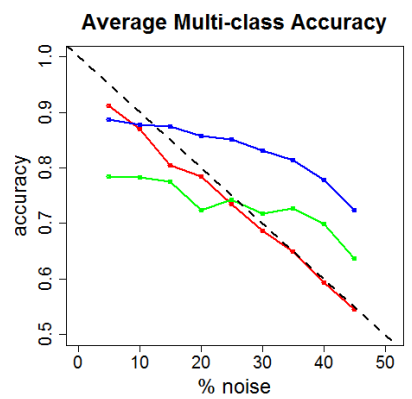

Fig. 13: Average accuracy results on multi-class data sets 

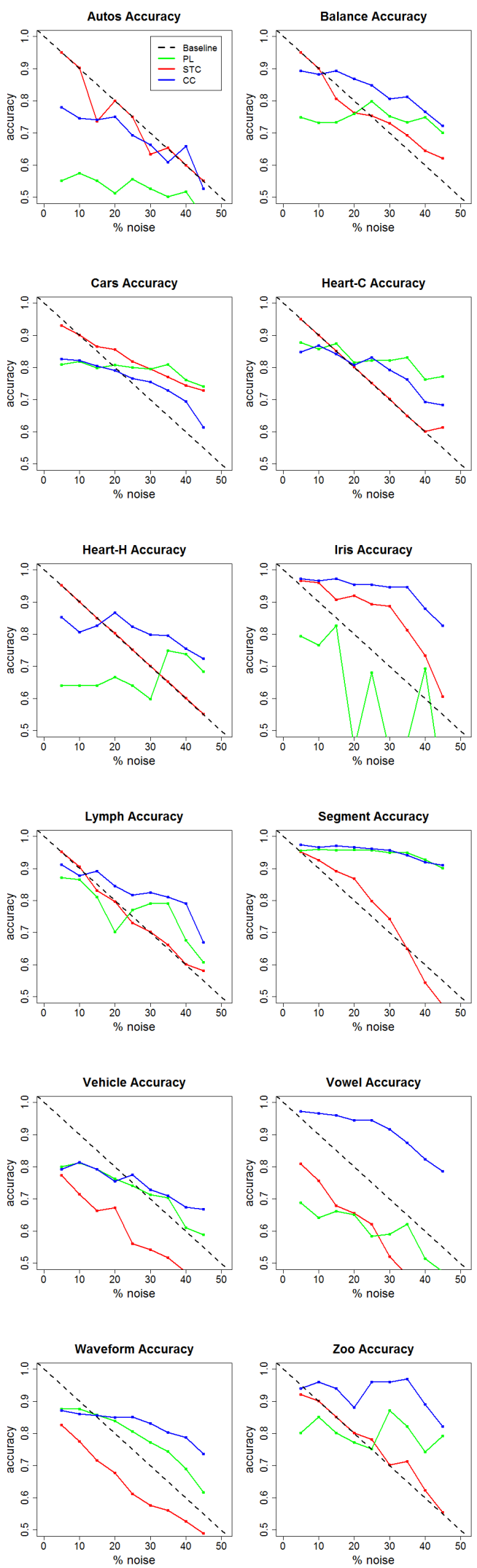

Fig. 10: Accuracy results on multi-class data sets. 

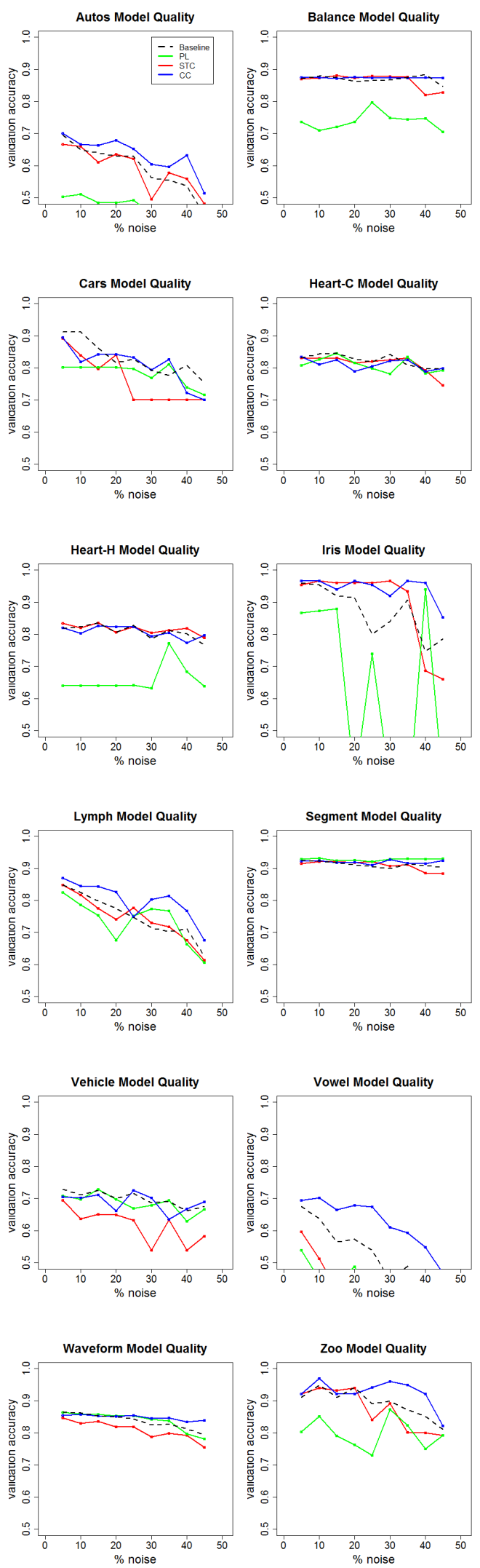

Fig. 11: Model quality results on multi-class data sets. 

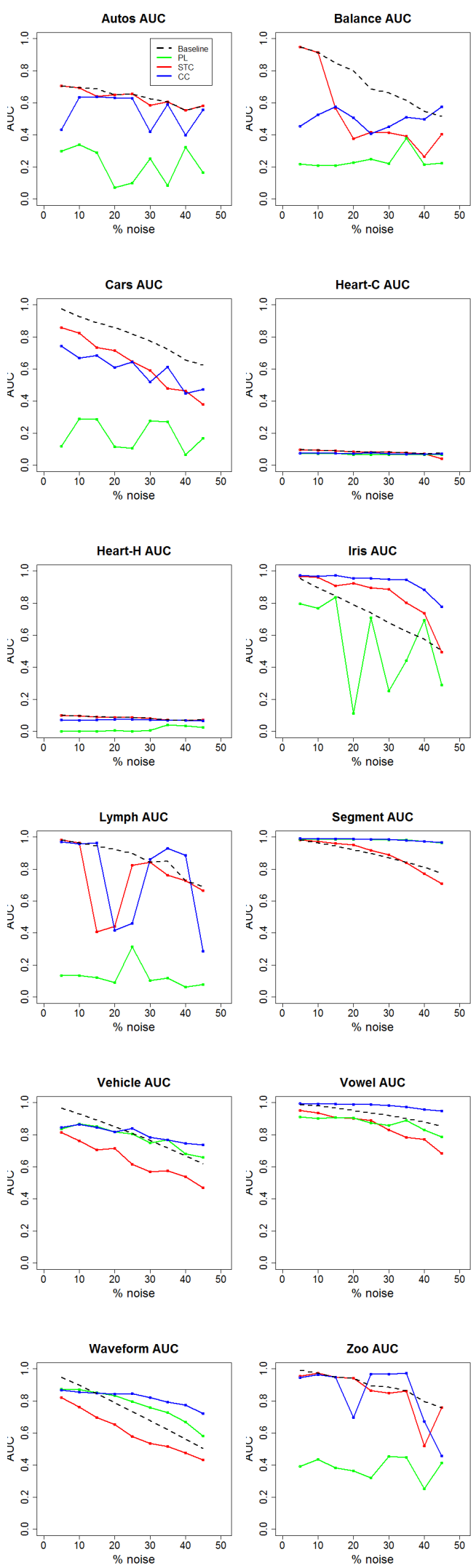

Fig. 12: AUC results on multi-class data sets. 


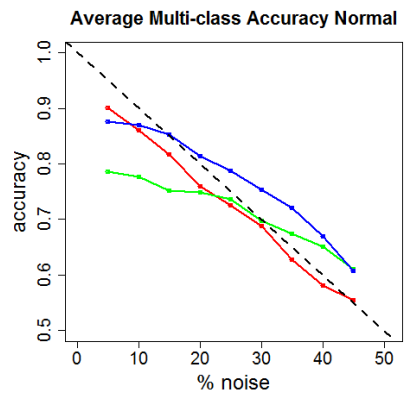

Fig. 14: Average accuracy results on multi-class data sets using normal distribution to generate noise

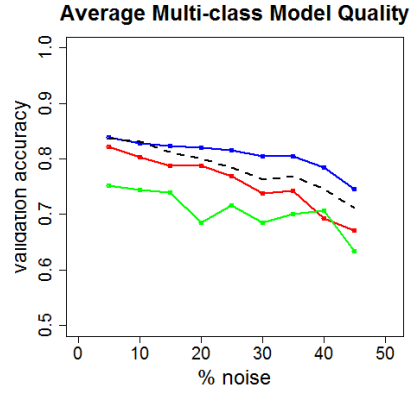

Fig. 15: Average model quality results on multi-class data sets

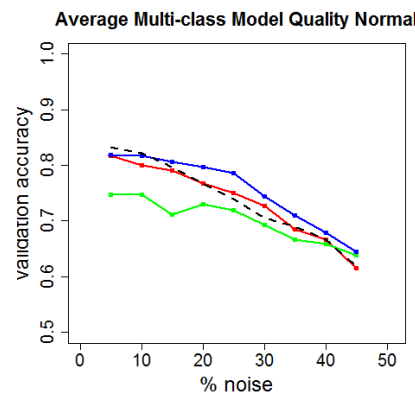

Fig. 16: Average model quality results on multi-class data sets using normal distribution to generate noise 


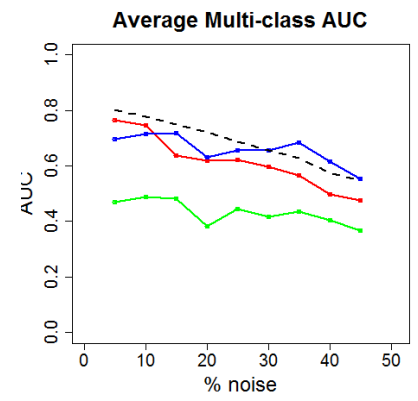

Fig. 17: Average AUC results on multi-class data sets

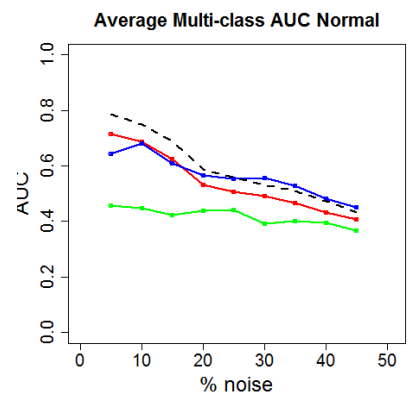

Fig. 18: Average AUC results on multi-class data sets using normal distribution to generate noise

Multi-class Data Sets The impact of the algorithms on accuracy of the 12 multi-class data sets is shown in Figure 10. The trends discussed for accuracy of the binary class data sets are a bit different for the multi-class data sets: $\mathrm{CC}$ performs the best, but it is PL, not STC, that is CC's competitor (in most cases). Both CC and PL perform much better (in terms of label quality) than STC on most of the multi-class data sets. STC does not work well on most of these data sets, except that it improves the label quality on the data sets iris and cars, and it also slightly improves the label quality on segment until the noise level $p \geq 0.35$. This is completely opposite from its performance on the binary data sets, where STC can almost always improve label quality. However, comparing the performance of both CC and PL on the binary data sets vs. the multi-class data sets, the label quality improvement of both CC and PL is much more significant on the multiclass data sets, especially under heavy noise. This observation is much more obvious if we compare their average performance on the binary data sets and on the multi-class data sets. 
Figure 11 shows the model quality improvements of each correction method on all twelve multi-class data sets. On the majority of these data sets, CC improves model quality much more than the other two methods (e.g. zoo, lymph, autos). STC generally is second-best in improving model quality, and PL is the worst. On some data sets (e.g. iris and heart- $h$ ), PL's resulting model quality is exceptionally poor.

Figure 12 shows the AUC improvements of the correction methods on the multiclass data sets. CC performs best (e.g. vowel, waveform, segment), STC shows decent performance, and PL is the worst. On most of the data sets, the correction methods can improve AUC from the baseline under some noise levels, but sadly, there were a couple of data sets (i.e. cars, autos) where the correction methods harmed AUC under all noise levels.

The average accuracy improvements of all three correction methods over the twelve multi-class data sets using uniform and normal noise generation are shown in Figure 13 and 14, respectively. CC is clearly superior to the other correction methods. CC can improve label quality when the noise level $p>0.10$ on average. PL cannot improve label quality until $p \geq 0.30$ on average, and STC cannot improve label quality at all, on average. Only on the noise level $p=0.05$ does STC outperform CC. Figure 13 clearly shows the trends discussed above: in the multi-class scenario, on the noise level interval $[0.25,0.45]$, PL outperforms STC.

Figures 15 and 16 show the average model quality improvements of the correction methods over the multi-class data sets. When the noise level $p \geq 0.15$, CC can improve model quality on average. Under uniform noise, STC and PL cannot improve model quality at all on average. However, under normally generated noise, STC improves model quality in the noise level interval [0.25, 0.30], and PL improves model quality when noise level $p=0.45$.

Finally, Figures 17 and 18 display the average AUC improvements of the three correction methods over the multi-class data sets. When the noise level $p \geq 0.35, \mathrm{CC}$ improves AUC on average, while the other two methods cannot improve AUC on any of the noise levels on average.

Insights Aside from the correction methods' performance relative to each other, there are other interesting observations to be made from these experimental results. All of the noise correction methods' results for accuracy, AUC, and model quality mirrored each other. That is, there was no trend of a correction method performing very well in accuracy, but doing poorly in model quality or AUC. This may be an indication that there exists a single metric that encapsulates all three of these measures (and perhaps more), or it may be simply an indication that if a correction method did well (or poorly) then it did so in general, not just in affecting one specific aspect of data quality. Another observation we can make is to highlight the clear differences between binary class and multi-class scenarios. The difference is so drastic that it essentially made two of the methods (PL and STC) switch roles (i.e., one did well while the other did poorly in one scenario, but in the other scenario, the reverse was true). This indicates that it may be necessary for the data practitioner to be aware of whether his data is specifically binary class or multi-class, and adjust his techniques accordingly. As a final observation, we note that it is very difficult, perhaps impossible, to improve data quality when there is 
very light noise. This suggests that performing noise correction at all comes at a risk, that risk being corrupting already clean data. Indeed, the line is thin between corruption and correction. However, noise correction can reverse much of the corruption of already-noisy data.

\section{Experiments on Crowdsourcing}

In order to evaluate the performance of the three methods in crowdsourcing, we conduct experiments on both real-world and synthetic crowdsourced data sets. For the synthetic data sets, we simulated crowdsourcing by creating ten "workers" to label each instance in the data set. Each worker has an accuracy in the interval $[0,1]$, and each instance has a bias, or a tendency to be answered as each class. The bias is represented as a distribution of values, one value for each class, summing to 1 . We modeled this bias using a probability distribution generated by WEKA's NaiveBayes classifier (Hall et al., 2009). From the worker's accuracy and the instance's bias, a probability distribution representing the likelihood that the worker will label the instance as any given class can be calculated. This probability distribution is calculated and the worker's label is chosen in the manner shown by Algorithm 3.

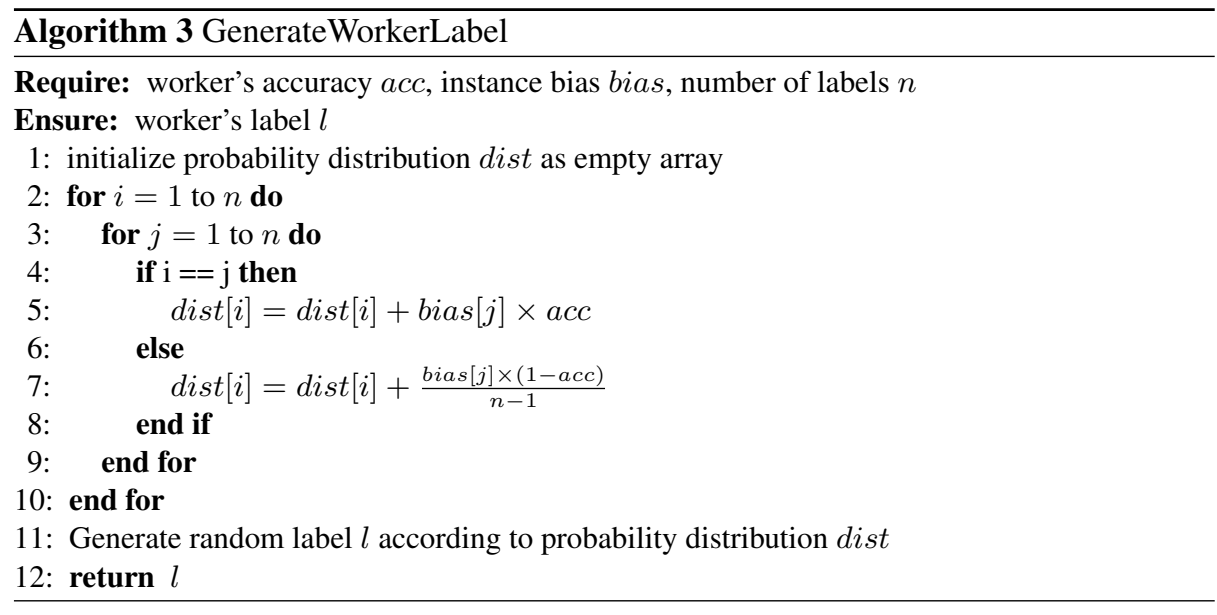

We compare the accuracy, which is the proportion of correctly labeled instances in the data set, achieved from majority voting consensus compared with DS and KOS. The performances of the three consensus algorithms (MV, DS, and KOS) serve as the baselines for the three correction methods (i.e., PL, STC, and CC), respectively.

All our data sets contain features extracted from images. Tables 3 and 4 show the data sets we used in our experiments: Table 3 shows the real-world crowdsourced data sets and Table 4 shows the synthetically crowdsourced data sets. All attributes in these data sets are numerical, because only numerical features were extracted from the images. 

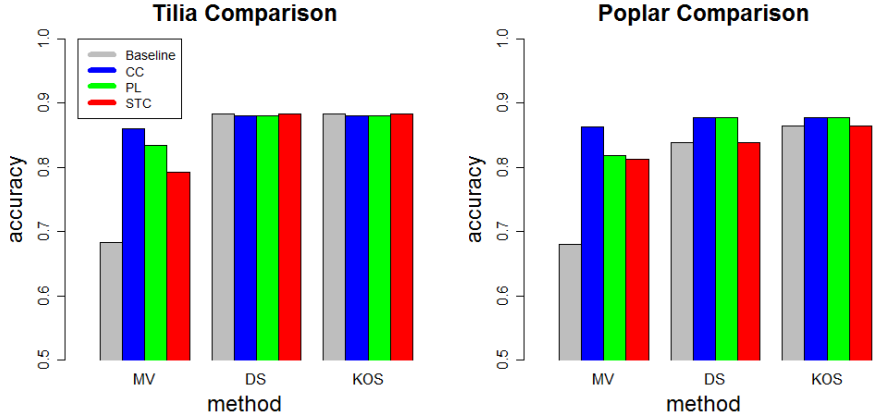

Fig. 19: Accuracy results for real-world data sets.
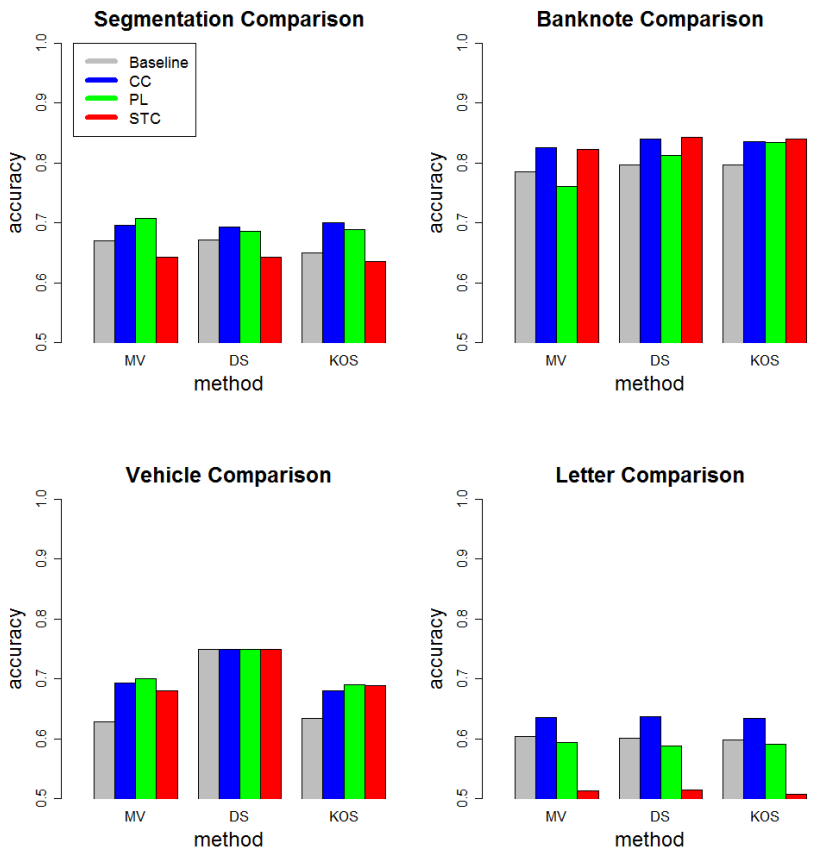

Fig. 20: Accuracy results for synthetic data sets.

\begin{tabular}{|l|l|l|l|}
\hline Data Set & \#Atts. & \#Instances & \#classes \\
\hline tilia & 64 & 384 & 2 \\
\hline poplar & 64 & 384 & 2 \\
\hline
\end{tabular}

Table 3: Real-World Data Sets. 


\begin{tabular}{|l|l|l|l|}
\hline Data Set & \#Atts. & \#Instances & \#classes \\
\hline segmentation & 19 & 2310 & 7 \\
\hline banknote & 4 & 1372 & 2 \\
\hline vehicle & 18 & 846 & 2 \\
\hline letter & 16 & 2600 & 26 \\
\hline
\end{tabular}

Table 4: Synthetic Data Sets.

The real data sets, tilia and poplar, each contain 384 images depicting a leaf. These images were posted on Amazon Mechanical Turk (AMT). For the tilia data set, the AMT workers were asked to label the image as containing a tilia leaf or not, and for the poplar data set, the workers were asked to label the image as containing a poplar leaf or not. For each image, ten labels were acquired.

The synthetic data sets, segmentation, banknote, vehicle and letter were found in the UC Irvine data set repository. Vehicle originally had four classes. In our experiments, we merge classes 2, 3, and 4, making it a binary class data set. Letter originally had 20,000 instances. We condense it by randomly selecting 100 instances from each class and discarding the rest.

Figure 19 shows the results obtained from inference and noise correction on the real-world crowdsourced data sets. On the data set tilia, DS and KOS both perform much better than MV. All three correction methods vastly improve MV's performance, with CC outperforming the other two. For DS and KOS, none of the correction methods could improve accuracy from their baselines. On the data set poplar, DS and KOS again dominate MV in label accuracy, with KOS slightly superior to DS. All the correction methods greatly improve MV's performance, as on the data set tilia. Again, CC performs the best. For DS and KOS, CC and PL are evenly matched, both of them improving accuracy from their baselines, while STC is even with its baselines.

Figure 20 shows the results from the synthetic data sets. There is no significant difference in performance among MV, DS, and KOS, except in vehicle, on which DS performs much better than the other two. Also, CC improves accuracy from its baselines almost every time, whereas PL does so a little over half the time, and STC does so less than half the time. CC's superiority is clearly shown on the data set letter.

Insights It is often the case, and especially so with CC, that regardless of the consensus method used before noise correction is performed, the correction method has the same performance. This seems to suggest that instead of striving to find an effective consensus method, it is just as effective to use the simplest consensus method (MV) and use noise correction instead. This has very wide implications, as it indicates that optimizing the consensus process may not be necessary or helpful when applying noise correction. It also implies that a sophisticated consensus method can be substituted with a noise correction method. Of course, more research on the combined effects of consensus and noise correction is necessary to solidify these conclusions. 


\section{Conclusions}

In this paper, we have set the groundwork for much more research on the topic of label noise correction, a topic that has been addressed much less than it deserves, given the importance of cleanly labeled data for the data practitioner. We have pointed out the lack of a formally defined label noise correction algorithm in literature. We proposed two novel label noise correction methods (CC and STC) and adapted one (PL). Our experimental results from the artificially noisy data sets showed that all three methods improve the label quality when the label noise reaches a certain level, and that only CC could reliably improve model quality and AUC. STC is second best in all scenarios except in improving label quality on mulit-class data sets, in which case it performs worst. We found that in all other scenarios, PL performs the worst among the three correction methods. Among the three methods, our proposed method CC performs the best on both binary class data sets and multi-class data sets, taking into consideration the three metrics we used for evaluation.

However, we also notice from the artificial noise level experiments that the three label noise correction methods could not improve the label quality further on half of the experimental data sets when the label noise level is lower than 0.10 . When the label noise is very low, it is very challenging to improve the label quality. We will conduct further research on this. As we know, data quality is important for information retrieval, statistics, and learning. An even more challenging quandary is why PL always harms model quality and AUC so greatly on multi-class data sets, while it improves the label quality when the noise level $p>0.25$. Future research is necessary to discover these answers.

Our experimental results on real-world and synthetically crowdsourced noisy data sets show that of the three consensus methods, KOS and DS seem to be the most effective. In terms of data quality improvement, CC performs the best overall, while PL does farily well and STC shows inconsistent results. Overall, the trend is an increase in accuracy of all the correction methods over the baselines, especially with MV as the baseline on crowdsourced data sets.

Potential future work topics abound. First, there are a few areas we could research further about our noise correction techniques. One undesirable result of our research was that the correction methods were harming data quality when the data sets had only light noise levels. It would be very beneficial to find a solution to this so that noise correction can be applied without fear of decreasing the data quality. Another area concerning the noise correction techniques that we could further research is a way to automatically select and tune parameters for correction (e.g., the threshold for Self-training Correction, the number of clusterers and initial centroids for each clusterer, etc.). Also, we could direct our research further toward the crowdsourcing field. For example, we could delve into why the more advanced consensus methods (i.e., not Majority Voting) paired with the noise correction methods had no different an effect than if either one were exclusively applied. Or more generally, we could see how our experimental results would differ if we consider not only image-based crowdsourced data sets but other tasks with features able to be used in machine learning. 


\section{Acknowledgements}

We thank the anonymous reviewers for the valuable comments. This research has been supported by the U.S. National Science Foundation under Grant No. IIS-1115417, the National Natural Science Foundation of China under Grant No. 61603186, the Natural Science Foundation of Jiangsu Province, China, under Grant No. BK20160843, and the China Postdoctoral Science Foundation under Grant No. 2016M590457.

\section{References}

Auer, P., \& Cesa-Bianchi, N. (1998). On-line learning with malicious noise and the closure algorithm. Annals of mathematics and artificial intelligence 23, no. 1-2, 83-99.

Brabham, D. C. (2008). Crowdsourcing as a model for problem solving an introduction and cases. Convergence: the international journal of research into new media technologies 14.1, 75-90.

Dawid, A. P., \& Skene, A. M. (1979). Maximum likelihood estimation of observer error-rates using the em algorithm. Applied Statistics, 20-28.

Devijver, J. K. (1980). On the edited nearest neighbor rule. In Proceedings of the fifth international conference on pattern recognition (p. 72-80).

Dietterich, T. G. (2000). An experimental comparison of three methods for constructing ensembles of decision trees: Bagging, boosting, and randomization. Machine learning 40, no. 2, 139-157.

Frenay, B., \& Verleysen, M. (2014). Classification in the presence of label noise: A survey. Neural Networks and Learning Systems, IEEE Transactions on, 25(5), 845-869.

Gaba, A., \& Winkler, R. L. (1992). Implications of errors in survey data: a bayesian model. Management Science 38, no. 7, 913-925.

Gamberger, D., Boskovic, R., Lavrac, N., \& Groselj, C. (1999). Experiments with noise filtering in a medical domain. In Proceedings of the 16th international conference on machine learning (p. 143-151).

Hall, M., Frank, E., Holmes, G., Pfahringer, B., Reutemann, P., \& Witten, I. H. (2009). The weka data mining software: an update. ACM SIGKDD explorations newsletter 11, no. 1, 10-18.

Jain, A. K., Murty, M. N., \& Flynn, P. J. (1999). Data clustering: a review. ACM computing surveys (CSUR) 31, no. 3, 264-323.

Kamar, E., Hacker, S., \& Horvitz, E. (2012). Combining human and machine intelligence in large-scale crowdsourcing. In Proceedings of the 11th international conference on autonomous agents and multiagent systems-volume 1 (p. 467-474). International Foundation for Autonomous Agents and Multiagent Systems.

Karger, D. R., Oh, S., \& Shah, D. (2011). Iterative learning for reliable crowdsourcing systems. In Advances in neural information processing systems (p. 1953-1961).

Khoshgoftaar, T., \& Rebours, P. (2007). Improving software quality prediction by noise filtering techniques. J. Comput. Sci. Technol. 22, 387-396. 
Kittur, A., Chi, E. H., \& Suh, B. (2008). Crowdsourcing user studies with mechanical turk. In Proceedings of the sigchi conference on human factors in computing systems (p. 453-456). ACM.

Likas, A., Vlassis, N., \& Verbeek, J. J. (2003). The global k-means clustering algorithm. Pattern recognition 36.2, 451-461.

Ma, T., Zhou, J., Tangi, M., Tian, Y., Al-Dhelaan, A., Rodhaan, M., et al. (2015). Social network and tag sources based augmenting collaborative recommender system. IEICE Transactions on Information and Systems 98, no. 4, 902-910.

Natarajan, N., Dhillon, I. S., Ravikumar, P. K., \& Tewari, A. (2013). Learning with noisy labels. In Advances in neural information processing systems (p. 11961204).

Nowak, S., \& Ruger, S. (2010). How reliable are annotations via crowdsourcing: a study about inter-annotator agreement for multi-label image annotation. In Proceedings of the international conference on multimedia information retrieval (p. 557-566). ACM.

Saez, J. A., Galar, M., Luengo, J., \& Herrera, F. (2014). Analyzing the presence of noise in multi-class problems: alleviating its influence with the one-vs-one decomposition. Knowledge and information systems 38, no. 1, 179-206.

Sanchez, J. S., Barandela, R., Marques, A. I., Alejo, R., \& Badenas, J. (2003). Analysis of new techniques to obtain quality training sets. Pattern Recognition Letters 24 , no. 7, 1015-1022.

Sheng, V. S., Provost, F., \& Ipeirotis, P. G. (2008). Get another label? improving data quality and data mining using multiple, noisy labelers. In Proceedings of the 14th acm sigkdd international conference on knowledge discovery and data mining (p. 614-622). ACM.

Song, Y., Wang, C., Zhang, M., Sun, H., \& Yang, Q. (2015). Spectral label refinement for noisy and missing text labels. In Twenty-ninth aaai conference on artificial intelligence (p. 2972-2978).

Tang, W., \& Lease, M. (2011). Semi-supervised consensus labeling for crowdsourcing. In Sigir 2011 workshop on crowdsourcing for information retrieval (cir).

Teng, C.-M. (1999). Correcting noisy data. In Proceedings of the sixteenth international conference on machine learning (p. 239-248). Morgan Kaufmann Publishers Inc.

Triguero, I., Saez, J. A., Luengo, J., Garcia, S., \& Herrera, F. (2014). On the characterization of noise filters for self-training semi-supervised in nearest neighbor classification. Neurocomputing 132, 30-41.

Wen, X., Shao, L., Xue, Y., \& Fang, W. (2015). A rapid learning algorithm for vehicle classification. Information Sciences 295, 395-406.

Wilson, M. E., Williams, N. B., Baskett, P. J., Bennett, J. A., \& Skene, A. M. (1980). Assessment of fitness for surgical procedures and the variability of anaesthetists' judgments.

Xia, Z., Wang, X., Sun, X., Liu, Q., \& Xiong, N. (2014). Steganalysis of lsb matching using differences between nonadjacent pixels. Multimedia Tools and Applications, 1-16.

Xia, Z., Wang, X., Sun, X., \& Wang, B. (2014). Steganalysis of least significant bit matching using multi-order differences. Security and Communication Networks 
7, no. 8, 1283-1291.

Xia, Z., Wang, X., Sun, X., \& Wang, Q. (2015). A secure and dynamic multi-keyword ranked search scheme over encrypted cloud data. IEEE Transactions on Parallel and Distributed Systems, (DOI: 10.1109/TPDS.2015.2401103).

Xu, R., \& Wunsch, D. (2005). Survey of clustering algorithms. Neural Networks, IEEE Transactions on 16, no. 3, 645-678.

Zheng, Y., Jeon, B., Xu, D., Wu, Q. M., \& Zhang, H. (2015). Image segmentation by generalized hierarchical fuzzy c-means algorithm. Journal of Intelligent and Fuzzy Systems: Applications in Engineering and Technology 28, no. 2, 961-973. 